\title{
Jugular Foramen Syndrome as Initial Presentation of Metastatic Lung Cancer
}

\author{
Dustin Hayward, M.D. ${ }^{1}$ Christopher Morgan, M.D. ${ }^{2}$ Bahman Emami, M.D. ${ }^{3}$ \\ Jose Biller, M.D., F.A.C.P., F.A.A.N., F.A.H.A. ${ }^{2}$ Vikram C. Prabhu, M.D., F.A.C.S. ${ }^{3}$ \\ 1 Department of Neurological Surgery, Loyola University Medical \\ Address for correspondence and reprint requests Vikram C. Prabhu, \\ Center, Maywood, Illinois \\ ${ }^{2}$ Department of Neurology, Loyola University Medical Center, \\ Maywood, Illinois \\ ${ }^{3}$ Department of Radiation Oncology, Loyola University Medical \\ Center, Maywood, Illinois \\ M.D., F.A.C.S., Associate Professor, Department of Neurological \\ Surgery, Loyola University Medical Center, 2160, South 1st Avenue, \\ Room 1900, Maguire Building, Maywood, IL 60153 \\ (e-mail: vprabhu@lumc.edu).
}

J Neurol Surg Rep 2012;73:14-18.

\begin{abstract}
Keywords

- skull base metastasis

- cranial nerve palsy

- Collet-Sicard syndrome

- soil and seed hypothesis

Metastatic involvement of the cranial base and jugular foramen generally presents with headache and lower cranial neuropathy but may escape early diagnosis. In this report, a patient developed a jugular foramen syndrome as the initial presentation of metastatic lung cancer soon after being diagnosed and treated surgically for extracranial atherosclerotic internal carotid artery disease. With the appropriate diagnosis established, he underwent local fractionated radiation therapy and systemic chemotherapy but succumbed to the disease. This report analyses metastatic disease affecting the cranial base and in particular, the jugular foramen, with a discussion of the clinical syndromes that accompany this rare condition.
\end{abstract}

Metastatic tumors involving the central nervous system (CNS) are common and occur in 20 to $40 \%$ of patients with a systemic malignancy. ${ }^{1-3}$ The frequency of intracranial metastases has increased in the recent years due to increased survival with better treatments of primary systemic disease and improved radiographic imaging. Common primary sources include lung, breast, and skin malignancies, and less frequently gastrointestinal, renal, prostate, testicular, and ovarian cancers. ${ }^{1,2,4-6}$ In up to $15 \%$ of patients, the primary site of malignancy is not known. ${ }^{1}$ Some neoplasms, such as melanoma, small-cell lung cancer, and choriocarcinoma, exhibit a unique "neurotropism," or propensity to spread to the CNS. ${ }^{4}$ Among children, the most common tumors that metastasize to the brain are neuroblastoma and sarcomatous tumors such as rhabdomyosarcoma, Ewing's sarcoma, and osteogenic sarcoma. ${ }^{3}$

Metastatic involvement of the cranial base may also be seen in $\sim 4 \%$ of patients with systemic malignancies. In particular, malignancies of the breast, lung, and prostate have a tendency to secondarily involve cranial base struc- tures. ${ }^{1,2,4-6}$ Most of these patients present with a cranial neuropathy. However, cranial neuropathy in a patient with systemic cancer may also be due to meningeal carcinomatosis or at times due to contiguous spread from a head and neck malignancy. ${ }^{2,7,8}$ In some instances, the presentation may be confusing and mimic a cerebrovascular, infectious, or metabolic affliction. In this unique case report, a patient developed a jugular foramen syndrome as the initial presentation of metastatic lung cancer soon after being diagnosed and treated surgically for extracranial atherosclerotic internal carotid artery (ICA) disease. The pathophysiology of cranial base metastases and the anatomical basis for the various cranial nerve syndromes that may result from this condition are discussed.

\section{Case Report}

This 70-year-old right-handed Caucasian man with a history of hypertension, hyperlipidemia, glaucoma, tobacco use, and a family history of lung cancer, presented to an outside facility received

September 8, 2011

accepted

September 23, 2011

published online

February 17, 2012
Copyright (C 2012 by Thieme Medical

Publishers, Inc., 333 Seventh Avenue, New York, NY 10001, USA.

Tel: +1(212) 584-4662.
Dol http://dx.doi.org/ 10.1055/s-0032-1301406. ISSN 2193-6358. 
with 1 week of headache and 2 days of difficulty articulating words with "speech changes." On examination, he had mild dysarthria, a left tongue deviation and a left Horner's syndrome. Computed tomography (CT) of the head was unremarkable except for age appropriate cortical atrophy. A magnetic resonance imaging (MRI) was not obtained during the workup as the patient reportedly had severe claustrophobia. A neurology consultation suggested that these changes were due to a left medullary brainstem stroke and a vascular etiology for the symptoms was suspected. A carotid ultrasound demonstrated 80 to $99 \%$ stenosis and a CT angiogram demonstrated $74 \%$ stenosis of the right ICA. A right carotid endarterectomy (CEA) was performed without complete resolution of his symptoms.

Three months later, he presented to the emergency department at the same outside facility with progressively worsening headache, dysarthria, inability to eat solids or expectorate his own oral secretions, lancinating left ear and left mastoid pain, decreased hearing, gait instability, vertigo, vomiting, and a $20-1 \mathrm{~b}$ weight loss. On examination, he was noted to have left-side hearing loss, uvular deviation to the right from a paretic left palate, a decreased left gag response, left tongue deviation, and a wide-based, unsteady gait ( - Fig. 1). MRI of the head demonstrated a left $2.5 \times 3.8 \times 4.0 \mathrm{~cm} \mathrm{T1/T2}$ hypointense, heterogeneously enhancing skull base mass involving the petrous apex, jugular bulb, cochlear aqueduct, hypoglossal canal, and occipital condyle ( - Fig. 2A, B). The mass had eroded into and thrombosed the left transverse-sigmoid sinus junction ( - Fig. 3 ). At this time, the patient was transferred to our institution for tertiary care.

On arrival, in addition to the above findings, we noted a hoarse voice with a left vocal cord paralysis along with atrophy of the left sternocleidomastoid, trapezius, and hypoglossal muscles suggesting a chronic process. A CT of the chest, abdomen, and pelvis demonstrated a right upper lobe pulmonary mass with mediastinal extension, enlarged hilar lymph nodes, and multiple hepatic lesions. A CT-guided biopsy of a hepatic lesion demonstrated nonsmall-cell lung

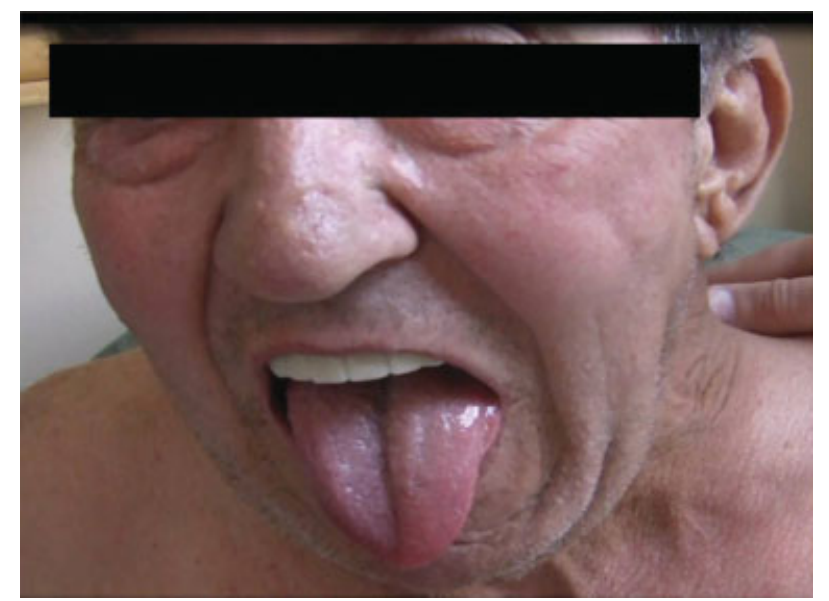

Figure 1 Patients showing cranial nerve XII involvement: left tongue atrophy and deviation to the left.

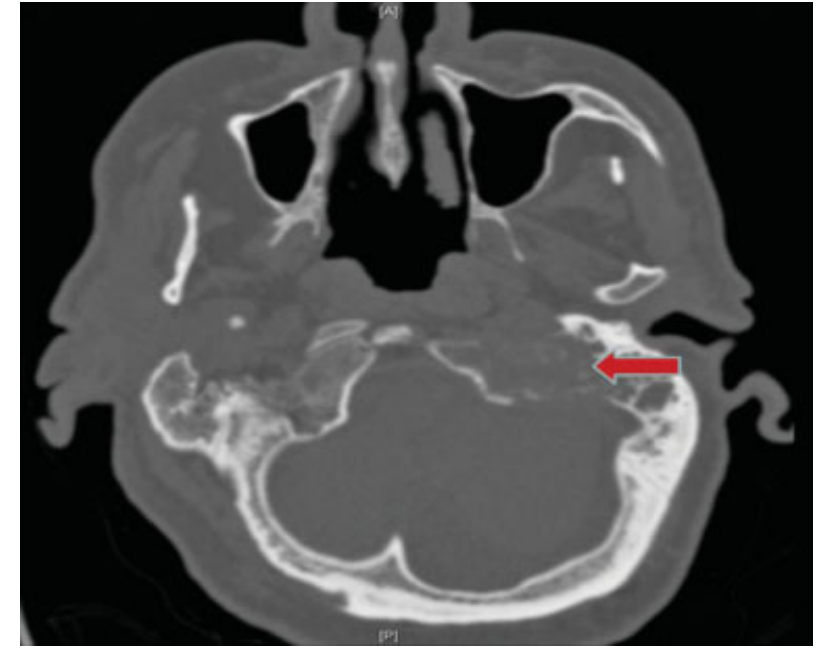

Figure 2 Cranial computed tomography scan demonstrating osteolytic destruction of the left petrous bone extending into the jugular foramen and occipital condyle.

adenocarcinoma. The patient was started on Navelbine (vinorelbine tartrate, Pierre Fabre Pharmaceuticals, France) chemotherapy and received $40 \mathrm{~Gy}$ in 10 fractions of palliative radiation therapy to the skull base lesion. Two months later, he developed an altered mental status and on repeat imaging was found to have watershed brain metastases. Due to his disease progression and poor performance status, the patient and family elected to pursue hospice care. He expired shortly thereafter.

\section{Discussion}

Intracranial metastases are the most common intracranial tumor and may be detected at the same time as the primary

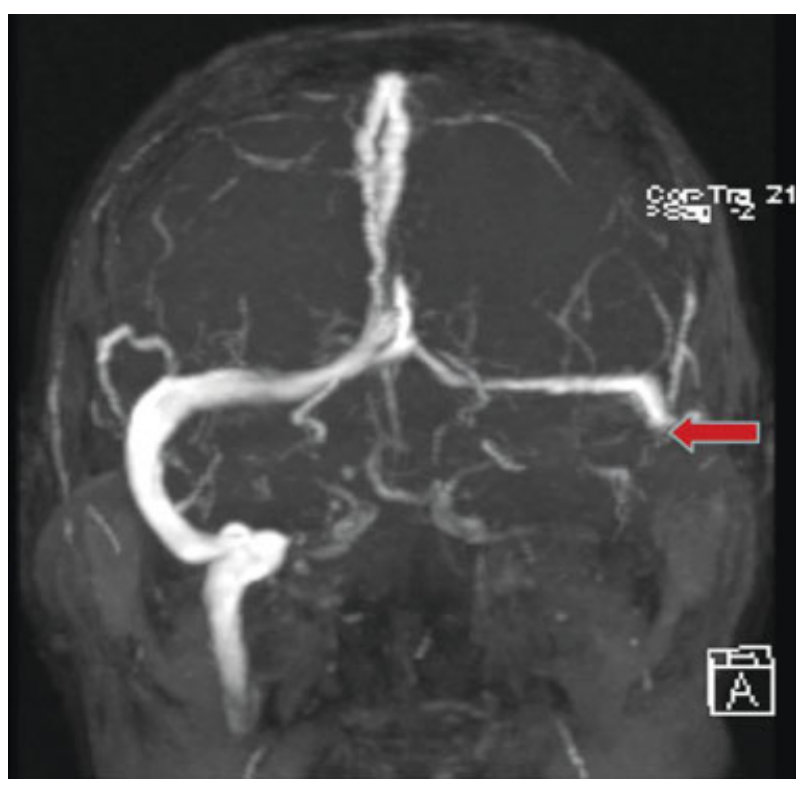

Figure 3 Magnetic resonance venogram demonstrating complete occlusion of the left transverse-sigmoid junction (arrow). 
malignancy in $\sim 30 \%$ of patients. $^{2}$ At times, they may be discovered with other sites of metastatic involvement in a metachronous fashion. In rare instances, they present in a precocious manner before evidence of the primary malignancy. ${ }^{2}$ Headache is the most common presenting symptom in patients with metastatic disease to the brain. In $40 \%$ of patients, a focal neurologic deficit is the presenting sign, while in 15 to $20 \%$, a seizure heralds the onset of the intracranial metastatic process. Papilledema is noted in 15 to $25 \%$ of patients and 5 to $10 \%$ of patients present with acute stroke-like symptoms which may be due to intratumoral hemorrhage, particularly with metastatic disease from melanoma, choriocarcinoma, or renal cell carcinoma. ${ }^{7}$ Significant vasogenic edema surrounding a metastatic lesion is common and is often associated with mass effect in some instances resulting in altered mental status or impaired cognition.

Metastatic disease involving the cranial base is generally considered a late event in the course of a systemic malignancy. However, metastatic disease is the most common cause of jugular foramen syndrome and is often underdiagnosed., ${ }^{4,9}$ Greenberg et al reported 36\% of all jugular foramen lesions were metastatic in origin with the most common primary lesions being breast, lung, kidney, and prostate cancers. ${ }^{10}$ Streitmann and Sismanis reported that a primary was discovered $67 \%$ of the time when a jugular foramen metastatic lesion is identified. In Greenberg's series, $~ 50 \%$ of patients had metastatic involvement elsewhere in the body before presenting with cranial base metastatic disease. ${ }^{11}$ LaigleDonadey et al reviewed 279 cases of skull base metastases and noted cranial base involvement as the first sign of cancer in $28 \%$ of patients. Prostate carcinoma represented the primary site of malignancy in $38 \%$; it was the most frequent cause of skull base metastasis in men, while breast carcinoma was the most frequent cause in women. Prostate cancer is known to metastasize to bone, particularly the vertebral bodies, pelvic bones, femurs, and ribs. ${ }^{12}$ Less commonly, it metastasizes to the cranial base, causing a variety of jugular foramen or cranial nerve dysfunction syndromes. Generally, this occurs in the face of metastatic prostate involvement of the axial skeleton, but there are rare reports of this cranial nerve dysfunction leading to the initial diagnosis of metastatic prostate cancer ${ }^{6}$; however, it is rarely an isolated occurrence and widespread metastatic disease is usually detected in these circumstances. ${ }^{6,9}$ Other neoplasms may also involve the cranial base in a metastatic fashion including malignancies of the colon, kidneys, thyroid, lymphoma, melanoma, and neuroblastoma.

Most intracranial metastatic disease is believed to arise from hematogenous spread from the primary tumor. ${ }^{2-4}$ Circulating tumor cells travel throughout the body and interact with the local environment at the molecular level. This results in the preferential lodging, invasion, and proliferation of specific tumors in certain organs. This mechanism of hematogenous spread is known as the "seed and soil" hypothesis. Embolization of tumor cells through the anterior circulation to terminal arteries in watershed areas at the graywhite matter junction are thought to give rise to intracranial metastatic disease. Hence, intracranial metastases are con- centrated where cerebral blood flow is greatest: $80 \%$ to the cerebral hemispheres, $15 \%$ to the cerebellum, and $5 \%$ to the brainstem. Other sites of involvement are rare. Two exceptions to this rule include melanoma and gastrointestinal tumors. Melanoma has a predilection to metastasize to the cerebral cortex and basal ganglia rather than the gray-white matter junction, while gastrointestinal tumors have a predilection to involve the cerebellum. ${ }^{5}$

Jugular foramen metastatic disease may not be the result of traditional hematogenous arterial spread. Rather, retrograde dissemination through Batson's plexus may be the route of hematogenous dissemination in these cases. ${ }^{4,9}$ Batson's plexus is a valveless venous plexus that connects abdominal structures with the cranial, spinal, and skull base through epidural and dural veins. This alternative mechanism of tumor metastasis may explain the common finding of metastasis at the thoracolumbar junction (T12-L2) and skull base via “seeding” by Batson's plexus. T2-weighted, T1-enhanced, fat suppression technique MRI is the best method to detect cranial base metastases. Radionuclide and CT scans are helpful as well in detecting lytic or osteoblastic lesions. Most metastatic lesions are lytic, but an osteoblastic mix pattern may be seen in prostate cancer primaries. Purely osteolytic lesions might be better detected by a study such as positron emission tomography, but in some instances single photon emission computed tomography scans have also been reported to have positive findings. ${ }^{10,13}$

Cranial base metastases may be clinically silent but frequently present with pain or cranial neuropathy. In fact, craniofacial pain in a patient who has systemic cancer is a significant finding. ${ }^{8}$ Laigle-Donadey et al identified five clinical syndromes of the cranial base secondary to metastatic disease. They termed them orbital, parasellar, middle fossa, jugular foramen, and occipital condyle syndromes. ${ }^{12}$ In their review, a predominance of the parasellar and sellar syndromes (29\%) was noted while middle fossa and jugular foramen syndrome were less common being seen in 6 and $3.5 \%$ of patients, respectively. In one-third of patients, the clinical symptomatology did not fit into one particular syndromic classification. Patients with jugular foramen metastases presented with hoarseness, dysphagia, and unilateral dull aching pain in the occipital and pharyngeal regions and behind the ear. On examination, they were noted to have involvement of CN IX through CN XII at times associated with Horner's syndrome.

The clinical syndromes associated with cranial base metastases in the region of the jugular foramen are best explained by understanding the local anatomy. The anterior surface of the jugular foramen is formed by the petrous temporal bone and posterior surface by the occipital bone. It is anatomically divided into three compartments, two venous and one neural. ${ }^{11}$ The large, laterally located venous compartment houses the sigmoid sinus and jugular bulb. The small, medially located venous compartment contains the inferior petrosal sinus. The neural compartment is located between the two vascular compartments. It contains the glossopharyngeal (IX), vagus (X), and accessory (XI) cranial nerves which all originate from a descending line of rootlets 
in the postolivary sulcus of the brainstem. Additional nervous structures passing within the foramen include the tympanic branch of the glossopharyngeal nerve (Jacobson's nerve) and the auricular branch of the vagus nerve (Arnold's nerve). Arterial structures passing through the foramen include the meningeal branch of the ascending pharyngeal artery and

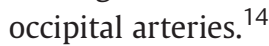

The glossopharyngeal nerve (IX) is primarily sensory with a small motor component. It receives general visceral and special sensory input from many structures, including the tonsils, larynx, middle ear, posterior one-third of the tongue, and carotid bodies. It projects a small general motor efferent to the stylopharyngeus muscle, which aids in the elevation of the larynx and pharynx. The vagus nerve $(\mathrm{X})$ is diverse in function and contributes to heart rate regulation, peristalsis, sweating, speech phonation, and elevation of the palate. The spinal accessory nerve (XI) is a pure motor efferent innervating the ipsilateral sternoclidomastoid and trapezius muscles. $^{15}$

Jugular foramen syndrome, or Vernet's syndrome, is characterized by dysfunction of the IX, X, and XI cranial nerves. ${ }^{3,8,15-17}$ Impingement of the vagus nerve at the level of the jugular foramen causes paralysis of the laryngeal muscles resulting in hoarseness and a nasal pitch. ${ }^{8,15-17}$ Further vagal compression results in unilateral paralysis of the soft palate and uvular deviation to the effected side. Involvement of the glossopharyngeal nerve results in a loss of sensation to the ipsilateral posterior tongue, decreased secretions from the ipsilateral parotid gland, and loss of the ipsilateral gag reflex. Dysfunction of the accessory nerve manifests as a shoulder droop, difficulty abducting the ipsilateral arm, and rotating the head contralaterally. Further, obstruction of the traversing sigmoid, jugular or inferior petrosal sinuses can result in intracranial venous congestion, cerebral edema, and intracranial hypertension which may manifest clinically as headache and papilledema. ${ }^{7,18}$ Clinical variations of the jugular foramen syndrome exist. Jackson's syndrome implies dysfunction of X, XI, and XII cranial nerves. Collet-Sicard syndrome consists of dysfunction of the lowest four cranial nerves (IX, X, XI, and XII), and when associated with an ipsilateral Horner's syndrome, it is known as Villaret's syndrome. Tapa's syndrome consists of X and XII nerves dysfunction., ${ }^{3,15}$ Although eponym heavy, this categorization scheme's ultimate purpose is to aid localization of skull base lesions.

Management of cranial base metastatic disease is complex and needs to be carefully individualized. Surgical resection may risk worsening cranial nerve deficits or cerebrovascular morbidity. Other complications include the risk of cerebrospinal fluid leakage or meningitis. Conformal fractionated radiation therapy to the region of involvement and control of the systemic malignancy with chemotherapy are frequently the best options. Radiation therapy provides relief of pain in up to $90 \%$ of patients and may also improve cranial nerve dysfunction with a salutary functional impact. Patients who present with symptoms of less than 1 month duration tend to do better following treatment with radiation therapy. The classic schedule of 30
Gy in 10 fractions is generally employed. Patients who have lymphoma and breast cancer might have a better response than patients who have prostate or lung cancer. In some instances, chemotherapy or hormonal therapy is employed in conjunction with radiation therapy that might have a positive impact on survival. Radiosurgery has been recently employed in the treatment of recalcitrant disease following primary radiation therapy. Complications with radiosurgery include worsening cranial nerve palsies, cerebral edema, or cerebrospinal fluid leakage. Status of the primary malignancy and the extent of systemic metastases determine mortality in these patients. ${ }^{19}$

In our report, we discuss a patient whose only initial presentation of metastatic lung cancer was most akin to Tapa's syndrome (cranial nerves X and XII palsy with Horner's syndrome), and later progressed to a Collet-Sicard syndrome with the additional involvement of cranial nerves IX and XI. The patient initially underwent a right CEA out of suspicion for carotid occlusion syndrome; however, his symptoms progressed as his skull base lesion expanded. The chronicity of his symptoms, and in particular, the atrophy of the involved muscles suggests a process that may have predated his carotid ischemic symptoms. This delay in diagnosis obviated treatment options including medical and radiation therapy that may have palliated the patient's symptoms and prolonged his survival.

\section{References}

1 Dichiro G, Fisher RL, Nelson KB. The jugular foramen. J Neurosurg 1964;21;447-460

2 Rhoton AL. Cranial Anatomy and Surgical Approaches. Hagerstown, MD: Lippincott Williams \& Wilkins2003

3 Graus F, Slatkin NE. Papilledema in the metastatic jugular foramen syndrome. Arch Neurol 1983;40(13):816-818

4 Löwenheim H, Koerbel A, Ebner FH, Kumagami H, Ernemann U, Tatagiba M. Differentiating imaging findings in primary and secondary tumors of the jugular foramen. Neurosurg Rev 2006; 29(1):1-11, discussion 12-13

5 Osborne AG. Diagnostic Neuroradiology. Philadelphia, PA: Mosby Inc. 1994

6 McDermott RS, Anderson PR, Greenberg RE, Milestone BN, Hudes GR. Cranial nerve deficits in patients with metastatic prostate carcinoma: clinical features and treatment outcomes. Cancer 2004;101(7):1639-1643

7 Bone I, Hadley DM. Syndromes of the orbital fissure, cavernous sinus, cerebellopontine angle, and skull base. J Neurol Neurosurg Psychiatry 2005;76(Suppl III):29-38

8 Robbins KT, Fenton RS. Jugular foramen syndrome. J Otolaryngol 1980;9(6):505-516

9 Carroll CG, Campbell WW. Multiple cranial neuropathies. Semin Neurol 2009;29(1):53-65

10 Greenberg HS, Deck MD, Vikram B, Chu FC, Posner JB. Metastasis to the base of the skull: clinical findings in 43 patients. Neurology 1981;31(5):530-537

11 Streitmann MJ, Sismanis A. Metastatic carcinoma of the temporal bone. Am J Otol 1996;17(5):780-783

12 Laigle-Donadey F, Taillibert S, Martin-Duverneuil N, Hildebrand J, Delattre JY. Skull-base metastases. J Neurooncol 2005;75(1): 63-69

13 Schmidt F, Dihné M, Steinbach J, Bühring U, Küker W. [Raeder- and Collet-Siccard-Syndrome. Acute pareses of cranial nerves 
18 Jugular Foramen Syndrome Hayward et al.

symptomatic of a dissection of internal carotid artery]. Nervenarzt 2000;71(6):502-505

14 Greenberg MS. Handbook of Neurosurgery. New York: Thieme Medical Publishers; 2006

15 Biller J, Brazis PW, Masdeu JC. Localization in Clinical Neurology. Hagerstown, MD: Lippincott Williams and Wilkins; 2011

16 Shine NP, O'Sullivan P. Collet-Sicard syndrome: a rare presentation of metastatic prostate adenocarcinoma. Auris Nasus Larynx 2005;32(3):315-318
17 Agarwal A, Baisakhiya N, Kakani A, Bhake A, Nagrale M, Reddy S. Metastatic lung cancer presenting with jugular foramen syndrome in a case of von Recklinghausens disease. J Cancer Res Ther 2010;6 (3):391-393

18 Schweinfurth JM, Johnson JT, Weissman J. Jugular foramen syndrome as a complication of metastatic melanoma. Am J Otolaryngol 1993;14(3):168-174

19 Soffietti R, Rudā R, Mutani R. Management of brain metastases. J Neurol 2002;249(10):1357-1369 